Research Paper

\title{
Effects of Tilting Mechanism of Narrow Vehicle on Psychophysiological States of Driver
}

\author{
Jongseong Gwak ${ }^{1)}$ Junsu Cho $^{1)}$ Keizo Araki ${ }^{1)}$ Toshiyuki Sugimachi ${ }^{2)}$ \\ Noboru Kubo ${ }^{1)}$ Yoshihiro Suda ${ }^{1)}$ \\ 1) The University of Tokyo, Institute of Industrial Science \\ 4-6-1 Komaba, Meguro, Tokyo, 153-8505, Japan (E-mail: js-gwak@iis.u-tokyo.ac.jp) \\ 2) Tokyo City University, Department of Industrial Science \\ 1-28-1 Tamatsutsumi, Setagaya, Tokyo, 158-8557, Japan
}

Received on March 25, 2020

\begin{abstract}
Narrow tilting vehicles have been proposed to address transportation issues such as traffic congestion, lack of parking space. The investigation of the effects of narrow tilting vehicles on user is insufficient though many methods for improving the stability of those were proposed. The purpose of the present study is to investigate the effects of tilting mechanism of narrow vehicles on psychophysiological states of driver as a fundamental study. Focused on user satisfaction among the components of usability, the hypotheses that a tilting mechanism affects the user's psychological state, and that the physiological indices such as a frontal alpha asymmetry, beta wave per alpha wave power based on brain activity are valid to evaluate the state were tested. The subjective evaluation of emotional states based on Russell's circumplex model and the measurement of electroencephalography (EEG) were performed in the experiment using the proposed vehicle with the tilting mechanism. As a result, both the subject evaluation and the physiological indices based on EEG showed a significantly higher value of arousal and valence in the case of the tilting vehicle compared to the control vehicle. These results suggest that both arousal and valence levels of narrow vehicle users can be improved by a tilting mechanism.
\end{abstract}

KEY WORDS: Human Engineering, Usability / Electroencephalography, Valence, Arousal, Narrow Tilting Vehicles [C2]

\section{Introduction}

In accordance with the increase in usage of private transport in urban areas ${ }^{(1)}$, transportation issues such as traffic congestion, fuel consumption, air pollution, and lack of parking space are expected to grow up. In order to address these issues, narrow vehicles have been developed with the expectation of the next urban transportation system (2). Considering the solution of the transportation issues, the narrower vehicle width would be better for space efficiency, but the vehicle width affects the stability of the vehicle. Therefore, narrow tilting vehicles have been proposed to maintain the stability of the vehicle body ${ }^{(3)}$. Previous studies were focused on a tilting mechanism to improve the stability ${ }^{(4)}$, and the building and evaluating of prototypes have been conducted (5). Our previous study proposed passive front-wheel steering that follows the vehicle body tilting for narrow track vehicles and investigated the steering stability of the vehicle ${ }^{(6)}$.

To address the transportation issues using these vehicles, not only the stability of the vehicle, but the improvement of usability such as driving comfort is also needed. However, the investigation of the usability of narrow tilting vehicles is insufficient though many methods for improving the stability of narrow track vehicles were proposed. Therefore, the present study purposed to investigate the usability of narrow tilting vehicles. It is defined that the usability consists of efficiency, effectiveness, and satisfaction in ISO ${ }^{(7)}$. As for the usability of narrow tilting vehicles, Matsuda et al. have investigated the adaptability of drivers to narrow tilting vehicles and easy to ride ${ }^{(8)}$. It seems that the adaptability of drivers in Matsuda's study is related to efficiency or effectiveness for driving. Sugiura et al. ${ }^{(9)}$ investigated the effects of a tilting function on the ride comfort using motion sickness incidence. In the present study, we focused on user satisfaction, which is defined in ISO as freedom from discomfort and positive attitude to the use of a product, while driving the narrow tilting vehicle. It was considered that user satisfaction is related to the psychological state of a driver from the definition. In addition, we considered that the psychological state of a driver may be significantly affected by the presence of the tilting mechanism since the degree of roll direction of a driver's body dynamically changed in accordance with the tilting of the vehicle body during driving. However, the effects of the tilting mechanism on the driver's psychological state were still unclear. To clarify the design requirements of the tilting mechanism, indices that can quantitatively evaluate the driver's psychological state are required. We considered that the physiological indices are appropriate for the quantitative evaluation of psychological state. The relationship between psychological states and electroencephalography (EEG), which is known as signals that reflect brain activities, has been investigated in previous studies. Alpha power of EEG is an inverse index of brain activity ${ }^{(10)}$. The asymmetry of the alpha power in frontal lobe is considered to reflect the balance between the right and left prefrontal activities (11), and it is associated with positive and negative affect ${ }^{(12,13)}$. In addition, beta power of EEG is an index of arousal ${ }^{(14)}$. Russell's circumplex model ${ }^{(15)}$, which is widely referred to psychological studies, treats the arousal and valence ('valence' means that the affective quality referring to the intrinsic 
attractiveness/goodness or averseness/badness of an event, object, or situation $\left.{ }^{(16)}\right)$ as an axis of the psychological state. Therefore, we focused on the EEG study for the quantitative evaluation of a psychological state.

The present study aimed to investigate the effects of the tilting mechanism on the psychophysiological state of driver while driving the narrow vehicle. The hypotheses that tilting mechanism affects the user's psychological state, and that the physiological indices such as a frontal alpha asymmetry, beta wave per alpha wave power based on EEG are valid to evaluate the state were tested using the proposed vehicle with a tilting mechanism.

\section{Materials and Methodology}

\subsection{Participants}

Eight males with a mean age of 36.6 years (SD 9.2 years) old and all right-handed participated in the experiment. Participants had been licensed drivers for a mean of 16.3 years (SD 11.3 years) in Japan. To reduce the effect on physiological responses, all participants were asked to sleep for six-hour or more and to avoid drinking and strenuous exercise on the day before the experiment.

\subsection{Experimental condition}

Eight conditions with the repeated-measures design were set in the experiment: 2 types of vehicle (control, tilting vehicle), 2 velocities of vehicle $(10,20 \mathrm{~km} / \mathrm{h}$ ), and 2 directions (clockwise, counterclockwise) of driving conditions were listed in Table 1 . The experiment was conducted in the ITS Field in the University of Tokyo. The driving course consisted of the straight sections of $20 \mathrm{~m}$ and the curved sections with the curvature radius of $8 \mathrm{~m}$, as shown in Figure 1. Driving for approximately $80 \mathrm{sec}$ was performed in each condition. The size of control vehicle and tilting vehicle is $2365 \times 955 \times 1600 \mathrm{~mm}$ and $1900 \times 650 \times 1500 \mathrm{~mm}$, and the weights of those are $290 \mathrm{~kg}$ and $250 \mathrm{~kg}$, respectively. Those have a similar operating system of steering and pedal with four wheels except for the presence of the tilting mechanism. A view of the control vehicle and the narrow vehicle with the tilting mechanism is shown in Figure 2.

\subsection{Measurement}

\subsubsection{Subjective evaluation}

To investigate the psychological state of drivers during each condition, participants were asked to provide detailed evaluations of an excited value, a nervous value, a bored value, and a relaxed value based on Russell's circumplex model ${ }^{(15)}$. An integer scale in the range from 1 to 5 was used to evaluate those four values.

\subsubsection{EEG recordings}

In order to extract the indices of valence and arousal referring to the brain activity, electrodes were attached to F3 and F4 channels based on the international 10-20 system of EEG measuring, as shown in Figure 3. These EEGs of the frontal lobe were recorded using biosignal instruments (Biosignalpux of PLUX, Portugal) at a sampling rate of $500 \mathrm{~Hz}$.

\subsection{Experimental procedure}

The experimental protocol was reviewed and approved by the Office for Life Science Research Ethics and Safety of the University of Tokyo. The detail of the experimental procedure was explained to the participants, and the experiment was conducted after obtaining informed consent from them. The participants were asked to performing a pre-driving for adaptation of operation and velocity control. After the pre-driving, the sensor for measuring EEGs was attached on the frontal lobe of participants. Finally, the main driving in each condition, as listed in Table 1, was performed. The questionnaire was performed by participants after each bout of main driving.

Table 1 Experimental conditions.

\begin{tabular}{|c|c|c|c|}
\hline Condition & Type of vehicle & Velocity $[\mathrm{km} / \mathrm{h}]$ & Direction of driving \\
\hline \hline 1 & Control & 10 & Clockwise \\
\hline 2 & Control & 10 & Counterclockwise \\
\hline 3 & Control & 20 & Clockwise \\
\hline 4 & Control & 20 & Counterclockwise \\
\hline 5 & Tilting vehicle & 10 & Clockwise \\
\hline 6 & Tilting vehicle & 10 & Counterclockwise \\
\hline 7 & Tilting vehicle & 20 & Clockwise \\
\hline 8 & Tilting vehicle & 20 & Counterclockwise \\
\hline
\end{tabular}

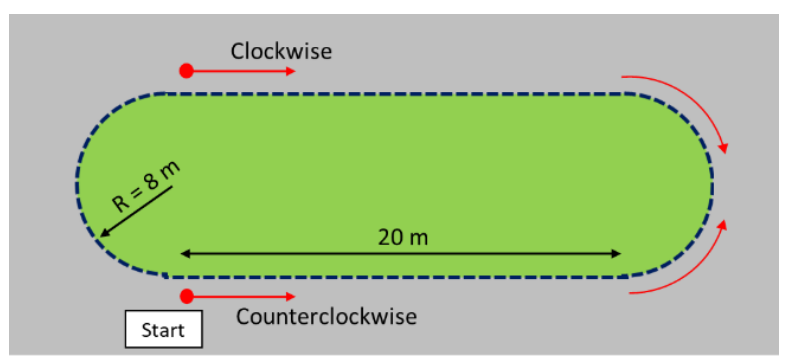

Fig. 1 The construction of driving course.
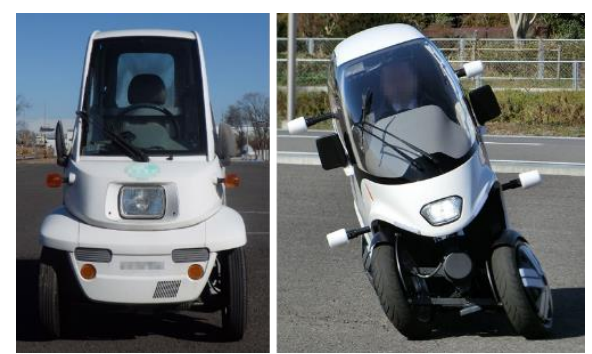

Fig. 2 A view of the control vehicle (left) and the narrow vehicle with a tilting mechanism (right).

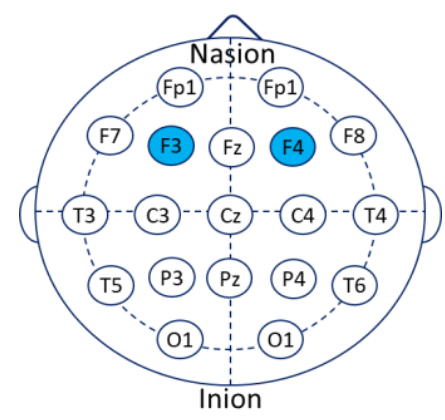

Fig. 3 Locations of F3 and F4 channel (the blue area) specified by the international 10-20 method. 


\subsection{Data processing and statistics}

The valence value and the arousal value were calculated from the evaluated points of each question by referring to Egawa's the estimation method of emotional centroid (17), which is based on Russell's circumplex model of affect ${ }^{(15)}$. Each evaluated point was plotted on the axis of Russell's circumplex model, as shown in Figure 4. The positions of the four points (excited, nervous, bored, and relaxed) were also decided regarding the positions of that in Russell's model. The state point was plotted by calculating barycentric coordinates of four points on the coordinate plane. Finally, valence value and arousal value were obtained from the $\mathrm{x}$, $\mathrm{y}$ coordinate of the state point, respectively.

Raw signal data of EEGs were processed using the MATLAB toolbox (Mathworks, USA) to remove the noise signals derived from blinks, body movements, and heartbeat ${ }^{(18,19)}$ based on bandpass filtering $(2 \sim 30 \mathrm{~Hz})$ and manual processing. Next, the power spectral density (PSD) of alpha wave $(8 \sim 13 \mathrm{~Hz})$ and beta wave $(13 \sim 30 \mathrm{~Hz})$ was calculated using the fast Fourier transform (FFT) method. Finally, the frontal alpha asymmetry (FAA) was calculated using Equation (1) based on the difference between left and right PSD of alpha and beta wave as the index of valence ${ }^{(20,21)}$. A high value of this index means positive valence. In addition, $\beta / \alpha$ was calculated using Equation (2) based on the PSD of the beta wave per the PSD of the alpha wave as the index of arousal ${ }^{(22,23)}$.

$$
\begin{aligned}
& F A \text { A value }=\frac{\alpha(F 4)}{\beta(F 4)}-\frac{\alpha(F 3)}{\beta(F 3)} \\
& \beta / \alpha \text { value }=\frac{\beta(F 3)+\beta(F 4)}{\alpha(F 3)+\alpha(F 4)}
\end{aligned}
$$

where $\alpha(i), \beta(i)$ is the PSD of alpha wave in location of $i$ and the PSD of beta wave in the location of $i$, respectively.

Finally, the Friedman test was used to compare each value between the 2 types of vehicle, velocity, and direction with a set of $95 \%$ confidence limits. The statistical analysis was performed with MATLAB.

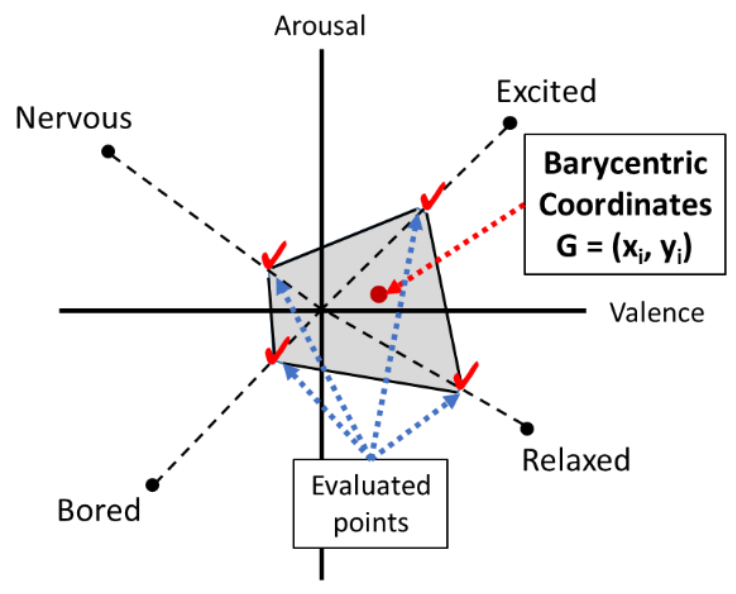

Fig. 4 Geometric diagram of the arousal value and the valence value adapted from the previous studies ${ }^{(15,17)}$.

\section{Results}

\subsection{Subjective evaluation}

The Friedman test was conducted on the valence value and the arousal value. The valence value in the case of tilting vehicle was significantly higher than that of the control vehicle $(p<0.001)$, as shown in Figure 5. In addition, the arousal value in the case of the tilting vehicle was also significantly higher than that of the control vehicle $(p=0.005)$, as shown in Figure 6 . As for the velocity and the direction, there were no significant differences in both the valence value and the arousal value between conditions.

\subsection{Physiological Indices}

The Friedman test was conducted on the FAA value and the $\beta / \alpha$ value. The FAA value in the case of the tilting vehicle was significantly higher than that of the control vehicle $(p=0.033)$, as shown in Figure 7. In addition, the $\beta / \alpha$ value in the case of the tilting vehicle was also significantly higher than that of the control vehicle $(p=0.003)$, as shown in Figure 8. Furthermore, the FAA value in the case of counterclockwise driving was significantly higher than that of clockwise driving $(p=0.012)$ as shown in Figure 9 . However, there was no significant difference between the $\beta / \alpha$ value in the case of counterclockwise driving and that of clockwise driving. When it comes to the velocity, there were no significant differences in both the valence value and the arousal value between conditions.

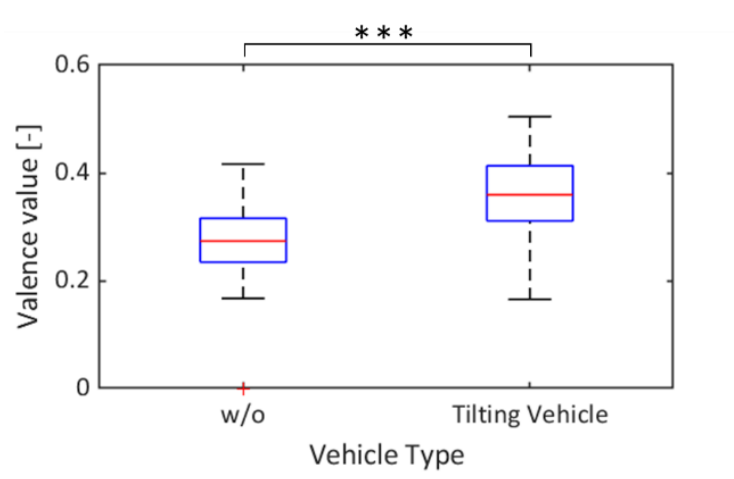

Fig. 5 Difference of the valence value between the case of control vehicle and tilting vehicle. (***: $p<0.001,95 \%$ confidence limits.)

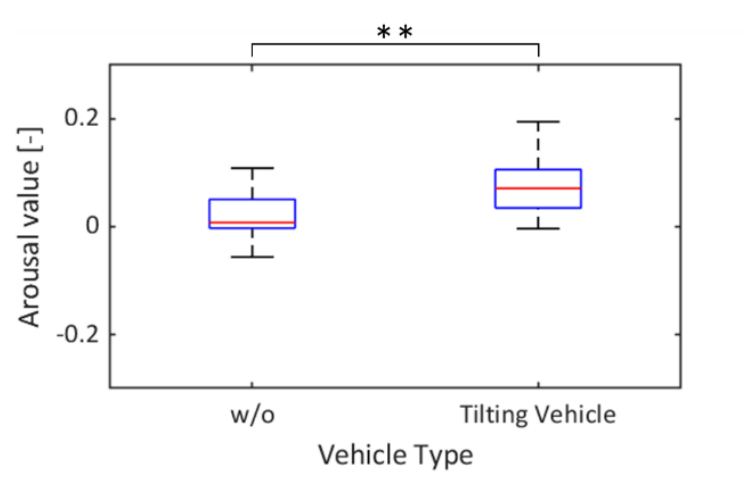

Fig. 6 Difference of the arousal value between the case of control vehicle and tilting vehicle.

(**: $p<0.01,95 \%$ confidence limits.) 


\section{Jongseong Gwak et al / International Journal of Automotive Engineering}

Vol.11, No.3(2020)

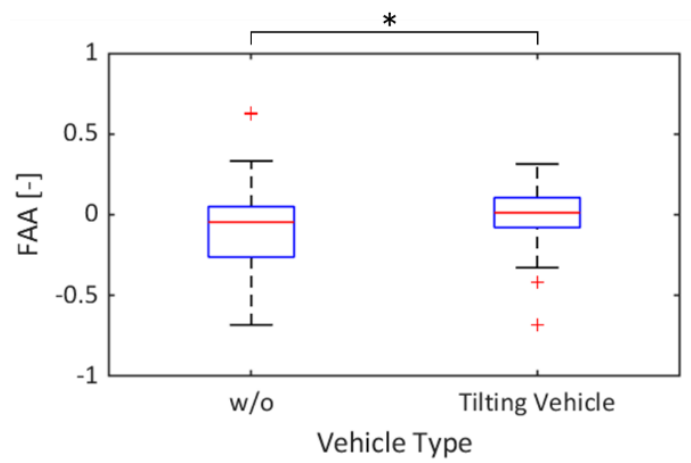

Fig. 7 Difference of the FAA value between the case of control vehicle and tilting vehicle.

$(*: p<0.05,95 \%$ confidence limits.)

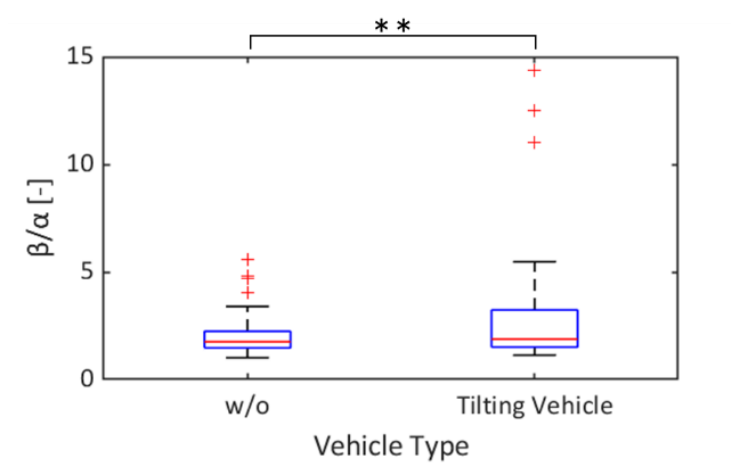

Fig. 8 Difference of the $\beta / \alpha$ value between the case of control vehicle and tilting vehicle. (**: $p<0.01,95 \%$ confidence limits.)

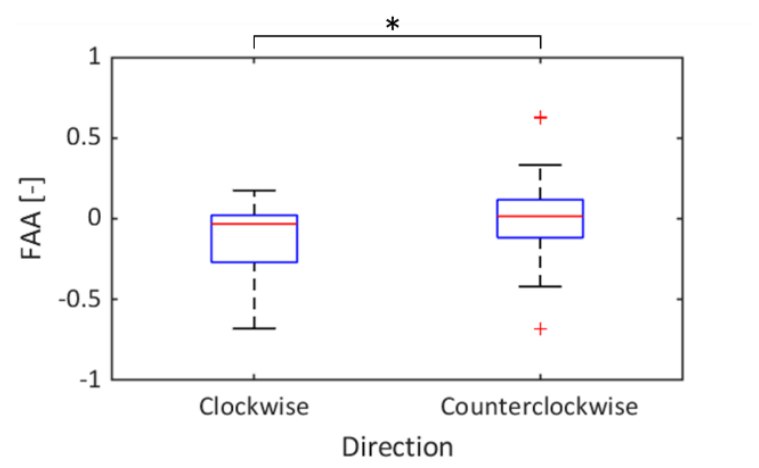

Fig. 9 Difference of the FAA value between the case of clockwise direction and counterclockwise direction.

$(*: p<0.05,95 \%$ confidence limits.)

\section{Discussion}

We tested the hypotheses that tilting mechanism affects the user's psychological state and that the physiological indices such as a frontal alpha asymmetry, beta wave per alpha wave power based on EEG are valid to evaluate the state. The main findings of the present study were that the tilting mechanism can increase both the arousal level and the valence level of driver significantly and that those significant differences can be evaluated by using the subjective evaluation, and the physiological indices based on EEG as well.
Our data indicated that the arousal level of users in the case of driving the tilting vehicle was higher than that of the control vehicle. A tilting mechanism causes the changes in the driver's posture in the external axis and the external view from the driver's eye-point. It is assumed that those changes may act as a stimulation to drivers, and thus the arousal level of driver may increase since these external stimulations impact to both visual sense and somatosensory. To clarify this mechanism, further quantitative data such as the driver's posture, head tilting, and eye-point are required. The lateral acceleration on drivers due to the centrifugal force while turning a curve is reduced by the tilting mechanism compared to the control vehicle. Thus it seems that the difference of the lateral acceleration while turning a curve between vehicles can cause positive valence in the case of the tilting vehicle. Sugiura et al. ${ }^{(9)}$ investigated the head toward the gravito-inertial force, head motion, and the relationship between head angular velocity and vehicle motion to evaluate the motion sickness. Those factors seem to affect the arousal and the valence of driver, thus the investigation of the relationship between those factors and psychophysiological indices is required to clarify the design requirement of tilting mechanism for improving user satisfaction, and that point is our future work.

The FAA has been used as the indices of valence in previous studies related to psychology ${ }^{(12,13,24)}$. However, the investigation of FAA in the case of a driving task was limited. Fairclough and Spiridon (25) investigated that the effects of traffic delays on psychophysiological activity using a driving simulator, and they thus showed that the index of FAA had a significant difference between the anger state due to the traffic delays and baseline levels. The present study supported their findings from the perspective of the feasibility of FAA to evaluate the psychological state in a driving task. In addition, the treatment of positive valence and the test with a real driving in the present study can reinforce that.

Our findings have implications for the optimization of the tilting system to improve user satisfaction. If the relationship between the system components of tilting and the indices used in the present study is clarified, the proper tilting system for a driver can be designed based on the finding. Furthermore, though the applications of FAA to evaluate driver's psychological states are limited in other vehicles, it seems that the FAA can be applied to evaluate the positive emotional states such as fun to drive.

The limitations of the present study should be discussed. First, the number of participants in the experiment was insufficient, and the age of them was limited. Moreover, the other factors except a tilting mechanism could not control completely since the proposed vehicle with a tilting mechanism has only a tilting mode when turning a curve. Further studies with the consideration of user variety and control variables are necessary to reinforce the validity, and these points are our future works.

\section{Conclusion}

The tilting mechanism of narrow vehicles suggests that it positively affects the driver's psychophysiological states. The evaluation methodology in the present study could be applied to optimize the tilting system for the improvement of user satisfaction and to evaluate the driver's psychological states in the case of other vehicles. However, further studies are needed to investigate the 
effects of user versatility. Future work will mainly investigate the relationship between the components of the tilting system and the driver's psychological states using the indices based on EEG.

This paper is written based on a proceeding presented at JSAE 2020 Annual Congress (Spring).

\section{References}

(1) Automobile Inspection \& Registration Information Association, Statistical Report of Vehicle Ownership in Japan (2019). https://www.airia.or.jp/publish/statistics/number.html (Accessed on 1 May 2020)

(2) R. Hibbard and D. Karnopp : Twenty first century transportation system solutions -a new type of small, relatively tall and narrow active tilting commuter vehicle, Vehicle Syst. Dyn., Vol.25, No.5, pp.321-347 (1996).

(3) S.G. So and D. Karnopp : Active dual mode tilt control for narrow ground vehicle. Vehicle System Dynamics, Vol.27 No.1, pp.19-36 (1997).

(4) C. Tang and A. Khajepour : Integrated stability control for narrow tilting vehicles: an envelope approach, IEEE Transactions on Intelligent Transportation Systems, pp.1-9 (2020).

(5) S. Maakaroun, W. Khalil, M. Gautier, and P. Chevrel : Modeling and simulating a narrow tilting car using robotics formalism, IEEE Transactions on Intelligent Transportation Systems, Vol.15, No.3, pp.1026-1038 (2014).

(6) J. T. C. Tan, H. Arakawa, Y. Suda, K. Araki, A. Mizuno, and M. Horiguchi : Steering stability of a passive front wheel design on tilting narrow track vehicle, in Proceedings of the 25th International Symposium on Dynamics of Vehicles on Roads and Tracks (IAVSD 2017), Vol.1 (2017).

(7) ISO/IEC. 9241-14 Ergonomic requirements for office work with visual display terminals (VDT)s - Part 14 Menu dialogues, ISO/IEC 9241-14: 1998 (E), (1998).

(8) M. Matsuda, I. Kageyama, Y. Kuriyagawa, T. Haraguchi, T. Kaneko, M. Kobayashi, and T. Murayama : Study on Driver Acceptability of Personal Mobility Vehicle with Lean Mechanism " , the Proceeding of 2018 JSAE Congress (Spring), No.20185123, pp. 1-6 (2018).

(9) T. Sugiura, T. Wada, T. Nagata, K. Sakai, and Y. Sato : Analysing Effect of Vehicle Lean Using Cybernetic Model of Motion Sickness, IFAC-PapersOnLine, No.52, Vol.19, pp.311316 (2019).

(10) I. A. Cook, R. O'Hara, S. H. J. Uijtdehaage, M. Mandelkern, and A. F. Leuchter : Assessing the accuracy of topographic EEG mapping for determining local brain function, Electroencephalography and Clinical Neurophysiology, Vol.107, No.6, pp.408-414 (1998).

(11) J. J. B. Allen, J. A. Coan, and M. Nazarian : Issues and assumptions on the road from raw signals to metrics of frontal EEG asymmetry in emotion. Biological Psychology, 67(1-2), pp.183-218 (2004).

(12) A. J. Tomarken, R. J. Davidson, R. E. Wheeler, and R. C. Doss : Individual differences in anterior brain asymmetry and fundamental dimensions of emotion, Journal of Personality and Social Psychology, Vol.62, No.4, pp.676-687 (1992).

(13) R. E. Wheeler, R. J. Davidson, and A. J. Tomarken : Frontal brain asymmetry and emotional reactivity: A biological substrate of affective style, Psychophysiology, Vol.30, No.1, pp.82-89 (1993).
(14) R. E. Dustman, R. S. Boswell, and P. B. Porter : Beta brain waves as an index of alertness, Science, Vol.137, No.3529, pp.533-534 (1962).

(15) A. Russell : A circumplex model of affect, Journal of Personality and Social Psychology, Vol.36, No.6, pp.11611178 (1980).

(16) N. H. Frijda, The Emotions. Cambridge(UK): Cambridge University Press, p. 207 (1986).

(17) S. Egawa, Y. Sejima, and Y. Sato : Proposal of an estimation method of emotional centroid based on the Russell's circumplex model for quantitative evaluation of affect, Transactions of Japan Society of Kansei Engineering (in Japan), Vol.18, No.3, pp.187-193 (2019).

(18) K. L. Coburn and M. A. Moreno : Facts and artifacts in brain electrical activity mapping, Brain Topogr., Vol.1, No.1, pp. 37 45 (1988).

(19) M. Fatourechi, A. Bashashati, R. K. Ward, and G. E. Birch : EMG and EOG artifacts in brain computer interface systems: A survey, Clinical Neurophysiology, Vol.118, No.3, pp.480494 (2007).

(20) J. A. Coan, J. J. B. Allen, and P. E. Mcknight : A capability model of individual differences in frontal EEG asymmetry, Biol. Psychol., Vol. 72, No. 2, pp.198-207 (2006).

(21) H. Jebelli, S. Hwang, and S. H. Lee : Feasibility of Field Measurement of Construction Workers' Valence Using a Wearable EEG Device, Computing in Civil Engineering 2017, pp.99-106 (2017).

(22) S. Giraldo and R. Ramirez : Brain-Activity-Driven RealTime Music Emotive Control, in Proceedings of the 3rd International Conference on Music \& Emotion, pp.11-15 (2013).

(23) T. McMahan, I. Parberry, and T. D. Parsons : Evaluating Player Task Engagement and Arousal Using Electroencephalography, Procedia Manufacturing, Vol.3, pp.2303-2310 (2015).

(24) R. Mennella, E. Patron, and D. Palomba : Frontal alpha asymmetry neurofeedback for the reduction of negative affect and anxiety, Behaviour Research and Therapy, Vol.92, pp.3240 (2017).

(25) S. H. Fairclough and E. Spiridon : Cardiovascular and electrocortical markers of anger and motivation during a simulated driving task, International Journal of Psychophysiology, Vol.84, pp.188-193 (2012). 